PROCEEDINGS OF THE

AMERICAN MATHEMATICAL SOCIETY

Volume 125, Number 12, December 1997, Pages 3605-3611

S $0002-9939(97) 04018-5$

\title{
A GENERALIZATION OF THE DE BRANGES THEOREM
}

\author{
XIE MING-QIN
}

(Communicated by Theodore W. Gamelin)

\begin{abstract}
In this paper a generalization of de Branges' proof of the Bieberbach conjecture is given. The argument does not make use of the Askey-Gasper theorem.
\end{abstract}

\section{INTRODUCTION}

It is well known that in 1984 the American mathematician de Branges [1], [2] proved the famous Bieberbach conjecture, thus, solving completely a problem that had challenged many mathematicians over a period of seventy years.

In this paper, the author gives a generalization of de Branges' proof. The new methods used are quite elementary and yield de Branges' result without using the Askey-Gasper theorem [3].

\section{Result}

In this section we shall give the main theorem and its proof.

Theorem. Let

$$
f(z)=z+\sum_{n=2}^{\infty} a_{n} z^{n} \in S
$$

and

$$
\log \frac{f(z)}{z}=\sum_{k=1}^{\infty} C_{k} z^{k}, \quad|z|<1 .
$$

If for any real number set $\left\{\lambda_{k}\right\}_{k=1}^{n}, \lambda_{k} \geq 0(k=1,2, \ldots, n)$, the condition

$$
\lambda_{k}+2 \sum_{p=k+1}^{n}(-1)^{p-k} \lambda_{k} \geq 0
$$

is satisfied, then

$$
\sum_{k=1}^{n} k \lambda_{k}\left|C_{k}\right|^{2} \leq 4 \sum_{k=1}^{n} \frac{\lambda_{k}}{k}, \quad n=1,2, \ldots
$$

Equality holds if and only if $f(z)$ is a rotation of the Koebe function.

Received by the editors June 27, 1995 and, in revised form, July 9, 1996.

1991 Mathematics Subject Classification. Primary 30A32.

Key words and phrases. Univalent, Bieberbach conjecture, characteristic root.

(C) 1997 American Mathematical Society 
Corollary. In the theorem, set $\lambda_{k}=n-k+1(k=1,2, \ldots, n)$, then it becomes de Branges theorem.

Instead of the de Branges special function system, we consider the function system $S_{k}(t)(k=1,2, \ldots, n)$ defined by the following system of differential equations:

$$
\left\{\begin{array}{l}
\frac{S_{k+1}^{\prime}(t)}{k+1}+\frac{S_{k}^{\prime}(t)}{k}=S_{k+1}(t)-S_{k}(t) \\
S_{n+1}(t) \equiv 0 \\
S_{k}(0)=\lambda_{k}, \lambda_{k} \geq 0 \\
\quad(k=1,2, \ldots, n)
\end{array}\right.
$$

For a given set $\lambda_{k}(k=1,2, \ldots, n),(4)$ and (5) define a unique system of functions $\left\{S_{k}(t)\right\}_{k=1}^{n}$. Now we find expressions for the $S_{k}(t)$.

First, we can obtain from (4)

$$
\frac{S_{k}^{\prime}(t)}{k}=2 \sum_{m=k+1}^{n}(-1)^{m-k-1} S_{m}(t)-S_{k}(t) \quad(k=1,2, \ldots, n) .
$$

The characteristic equation of this system of differential equations is

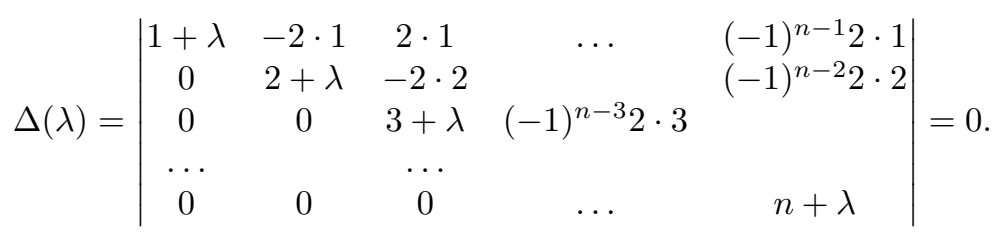

So its characteristic roots are

$$
\lambda_{1}=-1, \lambda_{2}=-2, \ldots, \lambda_{n}=-n .
$$

Hence we can suppose

$$
S_{k}(t)=\sum_{l=k}^{n} C_{k, l} e^{-l t} \quad(k=1,2, \ldots, n),
$$

where $C_{k, l}(l=k, k+1, \ldots, n)$ are constants.

Lemma 1. The functions $S_{k}(t)(k=1,2, \ldots, n)$ in (9) have forms

$$
S_{k}(t)=C_{k, k} e^{-k t}+\sum_{p=k+1}^{n} \alpha_{k, p} C_{p, p} e^{-p t}
$$

where $\alpha_{k, p}, C_{p, p}(p=k, k+1, \ldots, n)$ are constants and

$$
\begin{gathered}
\left\{\begin{array}{c}
\alpha_{k, p}=\frac{(-1)^{p-k} 2 k(2 p-1)(2 p-2) \cdots(p+k+1)}{(p-k) !} \\
(p=k, k+1, k+2, \ldots, n), \\
\alpha_{k, k}=1,
\end{array}\right. \\
\sum_{m=k}^{p}(-1)^{m-k} \alpha_{m, p}=\frac{(-1)^{p-k}(2 p-1)(2 p-2) \cdots(p+k)}{(p-k) !} .
\end{gathered}
$$


Proof. Substituting (9) in (6), we have

$$
S_{k}^{\prime}(t)+k S_{k}(t)=2 k \sum_{m=k+1}^{n}(-1)^{m-k-1}\left(\sum_{l=m}^{n} C_{m, l} e^{-l t}\right) .
$$

The solution of this differential equation is

$$
S_{k}(t)=C_{k, k} e^{-k t}+2 k \sum_{p=k+1}^{n}\left[\sum_{q=k+1}^{p}(-1)^{q-k} \frac{C_{q, p}}{p-k}\right] e^{-p t} .
$$

Therefore,

$$
C_{k, p}=\frac{2 k}{p-k} \sum_{q=k+1}^{p}(-1)^{q-k} C_{q, p} \quad(k=1,2, \ldots, n-1 ; p=k+1, k+2, \ldots, n) .
$$

From (13) it may be obtained that

$$
\begin{gathered}
C_{k, p}=\alpha_{k, p} C_{p, p}, C_{k+1, p}=\alpha_{k+1, p} C_{p, p}, \ldots, C_{p-1, p}=\alpha_{p-1, p} C_{p, p} \\
(k=1,2, \ldots, n-1 ; p=k+1, k+2, \ldots, n) .
\end{gathered}
$$

When $k=p-1,(13)$ is

$$
C_{p-1, p}=-2(p-1) C_{p, p}
$$

and $C_{p-1, p}-C_{p, p}=-(2 p-1) C_{p, p}$. Namely (11) and (12) are valid when $k=p-1$.

Now suppose (11) and (12) are valid if $k=m+1, m+2, \ldots, p-1$. Then by (13)

$$
\begin{aligned}
C_{m, p} & =-\frac{2 m}{p-m}\left(C_{m+1, p}-C_{m+2, p}+\cdots+(-1)^{p-m-1} C_{p, p}\right) \\
& =-\frac{2 m}{p-m} \cdot \frac{(-1)^{p-m-1}(2 p-1)(2 p-2) \cdots(p+m+1)}{(p-m-1) !} C_{p, p} \\
& =\frac{(-1)^{p-m} 2 m(2 p-1)(2 p-2) \cdots(p+m+1)}{(p-m) !} C_{p, p} .
\end{aligned}
$$

Hence (11) is true. Also,

$$
\begin{aligned}
C_{m, p}- & C_{m+1, p}+\cdots+(-1)^{p-m} C_{p, p} \\
= & {\left[\frac{(-1)^{p-m} 2 m(2 p-1)(2 p-2) \cdots(p+m+1)}{(p-m) !}\right.} \\
& \left.\quad-\frac{(-1)^{p-m-1}(2 p-1)(2 p-2) \cdots(p+m+1)}{(p-m-1) !}\right] C_{p, p} \\
= & \frac{(-1)^{p-m}(2 p-1)(2 p-2) \cdots(p+m)}{(p-m) !} C_{p, p},
\end{aligned}
$$

and (12) is true as well. This completes the proof of Lemma 1.

Lemma 2. For any positive integer $n=1,2, \ldots$, the identity

$$
\sum_{m=0}^{n}(-1)^{m} \frac{m^{k}}{m !(n-m) !}=0
$$

holds, where $k=0,1,2, \ldots, n-1$. 
Proof. By the binomial theorem

$$
(1-x)^{n}=n ! \sum_{m=0}^{n}(-1)^{m} \frac{x^{m}}{m !(n-m) !} .
$$

Apply the operator $x \frac{d}{d x}$ to the $(1-x)^{n} k$ times, then let $x=1$. This gives (14).

Lemma 3. In (10) the coefficients $C_{k, k}$ have the forms

$$
C_{k, k}=\sum_{p=k}^{n}(-1)^{p-k} \delta_{k, p} \lambda_{p} \quad(k=1,2, \ldots, n),
$$

where

$$
\begin{aligned}
\delta_{k, p} & =\left|\begin{array}{ccccc}
\alpha_{k, k+1} & \alpha_{k, k+2} & \ldots & \alpha_{k, p-1} & \alpha_{k, p} \\
1 & \alpha_{k+1, k+2} & \ldots & \alpha_{k+1, p-1} & \alpha_{k+1, p} \\
\ldots & & \ldots & & \\
0 & 0 & \ldots & 1 & \alpha_{p-1, p}
\end{array}\right| \\
& =\frac{(-1)^{p-k} 2 k(2 k+1)(2 k+2) \cdots(p+k-1)}{(p-k) !} \quad(p>k), \\
\delta_{k, k} & \equiv 1 .
\end{aligned}
$$

Proof. It follows by (5) and (10) that

$$
\left\{\begin{array}{cccc}
C_{1,1}+\alpha_{1,2} & C_{2,2}+\alpha_{1,3} & C_{3,3}+\cdots+\alpha_{1, n} & C_{n, n}=\lambda_{1}, \\
C_{2,2}+\alpha_{2,3} & C_{3,3}+\cdots+\alpha_{2, n} & C_{n, n}=\lambda_{2}, \\
\cdots & \cdots & \cdots & C_{n, n}=\lambda_{n} .
\end{array}\right.
$$

We then obtain from (17)

$$
\begin{aligned}
C_{k, k} & =\left|\begin{array}{ccccc}
\lambda_{k} & \alpha_{k, k+1} & \ldots & \alpha_{k, n-1} & \alpha_{k, n} \\
\lambda_{k+1} & 1 & \ldots & \alpha_{k+1, n-1} & \alpha_{k+1, n} \\
\ldots & & \ldots & & \\
\lambda_{n-1} & 0 & \ldots & 1 & \alpha_{n-1, n} \\
\lambda_{n} & 0 & \ldots & 0 & 1
\end{array}\right| \\
& =\sum_{p=k}^{n}(-1)^{p-k} \delta_{k, p} \lambda_{p} \quad(k=1,2, \ldots, n) .
\end{aligned}
$$

Calculating gives

$$
\begin{aligned}
\delta_{p-1, p} & =-\frac{2 p-2}{1 !} \\
\delta_{p-2, p-1} & =-\frac{2 p-4}{1 !}, \delta_{p-2, p}=\frac{(2 p-4)(2 p-3)}{2 !} .
\end{aligned}
$$

Suppose

$$
\begin{gathered}
\delta_{m, p}=\frac{(-1)^{p-m} 2 m(2 m+1)(2 m+2) \cdots(p+m-1)}{(p-m) !} \\
(m=k+1, k+2, \ldots, p-1 ; m<p \leq n) .
\end{gathered}
$$


Then by (16)

$$
\begin{aligned}
\delta_{k, p}= & \sum_{m=k+1}^{p}(-1)^{m-k-1} \alpha_{k, m} \delta_{m, p} \\
= & \sum_{m=k+1}^{p}(-1)^{p-m-1} \frac{2 k(2 m-1)(2 m-2) \cdots(m+k+1)}{(m-k) !} \\
& \quad \cdot \frac{2 m(2 m+1)(2 m+2) \cdots(p+m-1)}{(p-m) !} \\
= & \sum_{m=k+1}^{p}(-1)^{p-m-1} \frac{2 k(p+m-1)(p+m-2) \cdots(m+k+1)}{(m-k) !(p-m) !} .
\end{aligned}
$$

Let $m-k=\mu$; then (18) becomes

$$
\begin{aligned}
\delta_{k, p}= & \sum_{\mu=1}^{p-k}(-1)^{p-k-\mu-1} \frac{2 k(p+k+\mu-1)(p+k+\mu-2) \cdots(2 k+\mu+1)}{\mu !(p-k-\mu) !} \\
= & (-1)^{p-k} \frac{2 k(2 k+1)(2 k+2) \cdots(p+k-1)}{(p-k) !} \\
& \quad+\sum_{\mu=0}^{p-k}(-1)^{p-k-\mu-1} \frac{2 k(2 k+\mu+1)(2 k+\mu+2) \cdots(p+k+\mu-1)}{\mu !(p-k-\mu) !} .
\end{aligned}
$$

For fixed $p$ and $k$, the numerator of second term in the last formula is a $(p-k-1)$ th degree polynomial of $\mu$. It must be equal to zero by Lemma 2 . Hence

$$
\delta_{k, p}=(-1)^{p-k} \frac{2 k(2 k+1)(2 k+2) \cdots(p+k-1)}{(p-k) !} .
$$

Lemma 4. Let the functions $S_{k}(t)(k=1,2, \ldots, n)$ be defined by the system of differential equations (4), (5). Then

$$
-\frac{1}{k} S_{k}^{\prime}(0)=\lambda_{k}+2 \sum_{p=k+1}^{n}(-1)^{p-k} \lambda_{p} .
$$

Proof. It follows by the definition of $S_{k}(t)$ and (5) that

$$
-\frac{1}{n} S_{n}^{\prime}(0)=\lambda_{n}, \quad-\frac{1}{n-1} S_{n-1}^{\prime}(0)=\lambda_{n-1}-2 \lambda_{n} .
$$

Now suppose

$$
-\frac{1}{m} S_{m}^{\prime}(0)=\lambda_{m}+2 \sum_{p=m+1}^{n}(-1)^{p-m} \lambda_{p}
$$


when $m=k+1, k+2, \ldots, n$. However, by (10)

$$
\begin{aligned}
-\frac{1}{k+1} S_{k+1}^{\prime}(0) & =C_{k+1, k+1}+\sum_{p=k+2}^{n} \frac{p}{k+1} \alpha_{k+1, p} C_{p, p} \\
& =C_{k+1, k+1}+\sum_{p=k+2}^{n} \alpha_{k+1, p} C_{p, p}+\sum_{p=k+2}^{n} \frac{p-k-1}{k+1} \alpha_{k+1, p} C_{p, p} \\
& =\lambda_{k+1}+\sum_{p=k+2}^{n} \frac{p-k-1}{k+1} \alpha_{k+1, p} C_{p, p} .
\end{aligned}
$$

Therefore

$$
\sum_{p=k+2}^{n} \frac{p-k-1}{k+1} \alpha_{k+1, p} C_{p, p}=2 \sum_{p=k+2}^{n}(-1)^{p-k-1} \lambda_{p} .
$$

On the other hand, by (10)

$$
\begin{aligned}
-\frac{1}{k} S_{k}^{\prime}(0) & =C_{k, k}+\sum_{p=k+1}^{n} \frac{p}{k} \alpha_{k, p} C_{p, p} \\
& =C_{k, k}+\sum_{p=k+1}^{n} \alpha_{k, p} C_{p, p}+\sum_{p=k+1}^{n} \frac{p-k}{k} \alpha_{k, p} C_{p, p} \\
& =\lambda_{k}+\sum_{p=k+1}^{n} \frac{p-k}{k} \alpha_{k, p} C_{p, p} \\
& =\lambda_{k}-2 C_{k+1, k+1}+\sum_{p=k+2}^{n} \frac{p-k}{k} \alpha_{k, p} C_{p, p} .
\end{aligned}
$$

But we have

$$
\frac{p-k}{k} \alpha_{k, p}=\frac{(-1)^{p-k} 2(2 p-1) !}{(p-k-1) !(p+k) !}=-\frac{p+k+1}{k+1} \alpha_{k+1, p} .
$$

So from (21) and (22)

$$
\begin{aligned}
-\frac{1}{k} S_{k}^{\prime}(0) & =\lambda_{k}-2 C_{k+1, k+1}-\sum_{p=k+2}^{n} \frac{p+k+1}{k+1} \alpha_{k+1, p} C_{p, p} \\
& =\lambda_{k}-2\left(C_{k+1, k+1}+\sum_{p=k+2}^{n} \alpha_{k+1, p} C_{p, p}\right)-\sum_{p=k+2}^{n} \frac{p-k-1}{k+1} \alpha_{k+1, p} C_{p, p} \\
& =\lambda_{k}-2 \lambda_{k+1}-2 \sum_{p=k+2}^{n}(-1)^{p-k-1} \lambda_{p} \\
& =\lambda_{k}+2 \sum_{p=k+1}^{n}(-1)^{p-k} \lambda_{p} .
\end{aligned}
$$

Thus (19) is valid. 
Proof of the Theorem. We replace de Branges special system of functions by the special system of functions $\left\{S_{k}(t)\right\}_{k=1}^{n}$ and use his method to complete the proof.

\section{REFERENCES}

1. de Branges, A proof of the Bieberbach conjecture, Preprint E-5-84, Leningrad Branch of the V.A. Steklov Mathematical Institute, 1984.

2. __ A proof of the Bieberbach conjecture, Acta Math. 154 (1985), 137-152. MR 86h:30026

3. R. Askey and G. Gasper, Positive Jacobi polynomial sums. II, Amer. J. Math. 98 (1976), 709-737. MR 55:3363

Department of Mathematics, Anhui Normal University, Wuhu 241000, Anhui, People's Republic of China 